\title{
Exact cancellation of emittance growth due to coupled transverse dynamics in solenoids and rf couplers
}

\author{
David H. Dowell, Feng Zhou, and John Schmerge \\ SLAC National Accelerator Laboratory, 2575 Sand Hill Road, Menlo Park, California 94025, USA
}

(Received 25 July 2017; published 17 January 2018)

\begin{abstract}
Weak, rotated magnetic and radio frequency quadrupole fields in electron guns and injectors can couple the beam's horizontal with vertical motion, introduce correlations between otherwise orthogonal transverse momenta, and reduce the beam brightness. This paper discusses two important sources of coupled transverse dynamics common to most electron injectors. The first is quadrupole focusing followed by beam rotation in a solenoid, and the second coupling comes from a skewed high-power rf coupler or cavity port which has a rotated if quadrupole field. It is shown that a dc quadrupole field can correct for both types of couplings and exactly cancel their emittance growths. The degree of cancellation of the rf skew quadrupole emittance is limited by the electron bunch length. Analytic expressions are derived and compared with emittance simulations and measurements.
\end{abstract}

DOI: 10.1103/PhysRevAccelBeams.21.010101

\section{INTRODUCTION}

Optical aberrations are a major limitation to the beam quality of modern electron injectors and accelerators. This is especially true for the low-voltage guns and injectors required for high-duty factor and high-average current operation. In these systems, the beam is made large to mitigate space-charge forces. However, this large beam is more sensitive to aberrations such as the spherical aberration, which increases the emittance as the fourth power of the transverse beam size, and the chromatic aberration, which is proportional to the beam size squared.

Here we examine the coupled transverse dynamics aberration, which also strongly depends on the beam size. Coupled transverse dynamics results when the electrons have azimuthal momenta and their trajectories are no longer coplanar with the beam axis. The trajectories are "coupled" because the electron's $x$ coordinate depends not just on $x$ and $x^{\prime}$ but also on $y$ and $y^{\prime}$, and similarly for the $y$ coordinate. This paper assumes the mathematical theory is linear and applies $4 \times 4$-matrix algebra to compute the electron dynamics. We concentrate on the 4D transformation of a rotated quadrupole which skews the electrons about the beam axis. Since the 4D rotation is linear, the 4D emittance does not grow. However, there is emittance growth in both the $x-x^{\prime}$ and $y-y^{\prime}$ 2D phase spaces due to

\footnotetext{
*dowell@slac.stanford.edu
}

Published by the American Physical Society under the terms of the Creative Commons Attribution 4.0 International license. Further distribution of this work must maintain attribution to the author(s) and the published article's title, journal citation, and DOI. skew trajectories. Fortunately, the theory and simulation show that it can be eliminated with a rotated corrector field, because the coupling is correlated.

In his $1970 \mathrm{Ph} . \mathrm{D}$. thesis, Ripken described a theory of the coupled transverse dynamics in electron storage rings for high-energy physics experiments [1]. His and studies which followed [2] used 4D-matrix algebra to show that the beam luminosity could be increased (emittance decreased) by correcting for transverse plane coupling with a skew quadrupole. Recent theoretical work describes the coupled dynamics by generalizing the Courant-Snyder theory with a 4D symplectic rotation [3]. A useful introduction to the matrix theory for electron beams can be found in Wiedemann's book [4].

This paper concentrates on beam quality degradation due to quadrupole fields which are themselves rotated or when the beam has been rotated in a solenoid with respect to normally aligned quadrupole fields. Here we study two beam line components which are common sources of rotated quadrupole fields. The first is a weak quadrupole field followed by a solenoid, and the second is the quadrupole field produced by the coupler which feeds rf power into a cavity through rotated or unbalanced ports $[5,6]$.

The emittance of quadrupole followed by a solenoid is simply the emittance growth of the rotated quadrupole field, with a rotation angle equal to the sum of the quadrupole's rotation plus the beam's Larmor rotation in the solenoid. Although we assume the weak quadrupole field is near the solenoid, the field can, in fact, be anywhere before the solenoid. This includes the quadrupole $\mathrm{rf}$ fields of a rf gun followed by a focusing solenoid.

The transverse coupling due to rf fields can be eliminated by canceling the on-axis dipole and quadrupole fields with 
a dual rf feed and a racetracklike cavity shape [7]. Such designs have been implemented into modern rf guns [8] and upgraded rf couplers for room temperature linacs [9]. However, many accelerator rf cavities are built without these features, because the designs already exist, and the redesign, fabrication, and testing costs of new rf structures are prohibitively expensive, especially for superconducting rf cavities. Fortunately, as will be shown in this paper, much of this rework is unnecessary, since the quadrupole rf field can be exactly canceled with a low-field dc quadrupole.

This paper is organized as follows. The next section defines the terms rotated, normal, and skew quadrupole fields and derives the emittance growth produced by a rotated quadrupole field. Section III gives a general discussion of the fields of ideal solenoids, with only radial fields, and realistic solenoids possessing quadrupole fields. Quadrupole field measurements for a solenoid are presented. In Sec. IV, expressions for the coupled transverse dynamics emittance of the beam in a solenoid preceded by a weak quadrupole field are derived, and the emittance cancellation with a corrector quadrupole is demonstrated using analytic and numerical calculations as well as measurements. Section V discusses the rf quadrupole field, its induced emittance growth, and emittance cancellation with a dc corrector quadrupole. Finally, a summary of the work is presented in Sec. VI.

\section{EMITTANCE GROWTH DUE TO ROTATED, NORMAL, AND SKEW QUADRUPOLE FIELDS}

Figure 1 shows the equipotential surfaces for quadrupole magnetic fields with rotated, normal, and skew $B_{\theta}$-field patterns. The normal-quadrupole field (center drawing in Fig. 1) is aligned to the midplane of symmetry with $B_{y}(x, y)=-B_{y}(x,-y)$ and $B_{x}(x, y=0)=0$ along the $x$ axis. Rotating the field $45^{\circ}$ about the $+z$ axis (out of the page) results in a skew quadrupole field (right drawing in Fig. 1). The term rotated quadrupole is given to a quadrupole field having an arbitrary angle of axial rotation with respect to the normal-quadrupole field orientation. The rotated quadrupole field is equal to the vector sum of normal and skew quadrupole fields. As will be shown, only the skew component of a rotated quadrupole generates emittance, and therefore only a skew quadrupole corrector is necessary to cancel the emittance growth. The normal component of a rotated quadrupole produces no emittance; however, including a normal-quadrupole corrector allows returning the beam to its original transverse shape.

The beam transformation matrix for a quadrupole rotated about the $+z$ axis is found by first rotating the beam about the $z$ axis, then transforming through a normal quadrupole, and lastly rotating the beam back to zero rotation. Using the matrix and angle conventions of the TRANSPORT and MAD optics codes [10], the transformation matrix for a rotated quadrupole is given by

$$
R_{\text {rotquad }}(\alpha, f)=R_{\text {rot }}(-\alpha) R_{\text {quad }}(f) R_{\text {rot }}(\alpha) .
$$

Here $R_{\text {rot }}$ and $R_{\text {quad }}$ are the standard $4 \times 4$ matrices which rotate and quadrupole-focus the beam, respectively. $R_{\text {rot }}$ rotates the beam clockwise an angle $\alpha$ about the positive $z$ axis. Therefore, the quadrupole rotation angle $\alpha$ is shown going counterclockwise in Fig. 1. $R_{\text {quad }}$ is for a thin quadrupole lens with focal length $f$. Multiplying the matrices gives the transformation matrix for a thin quadrupole lens with focal length $f$ and rotation angle $\alpha$ as

$$
R_{\text {rotquad }}(\alpha, f)=\left(\begin{array}{cccc}
1 & 0 & 0 & 0 \\
\frac{-\cos 2 \alpha}{f} & 1 & \frac{-\sin 2 \alpha}{f} & 0 \\
0 & 0 & 1 & 0 \\
\frac{-\sin 2 \alpha}{f} & 0 & \frac{\cos 2 \alpha}{f} & 1
\end{array}\right) .
$$

The focal strength depends upon the beam energy and the integrated quadrupole field gradient:

$$
\frac{1}{f}=\left.\frac{e}{\beta \gamma m c} L_{\mathrm{eff}} \frac{\partial B_{y}}{\partial x}\right|_{x, y=0} .
$$
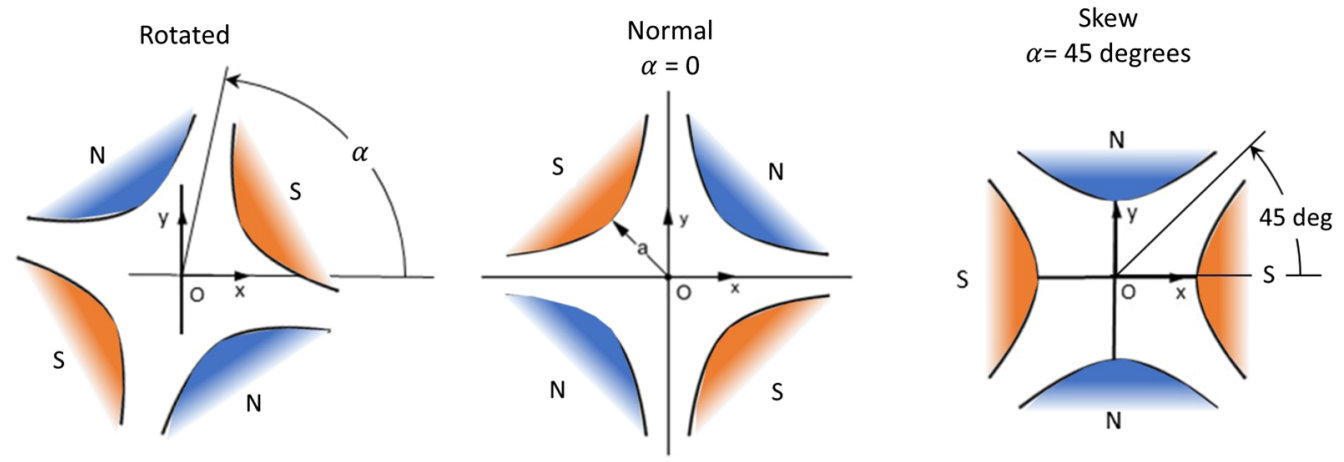

FIG. 1. Magnetic equipotential surfaces for rotated, normal, and skew quadrupole fields. The coordinate system is right-handed with the $z$ axis pointing out of the page. The normal-quadrupole field is focusing in the $x$ plane and defocusing in the $y$ plane for electrons traveling along the $+z$ axis. 
Here the effective length of the quadrupole field is $L_{\text {eff }}$, $\beta \gamma m c$ is the beam momentum, and $\left.\frac{\partial B_{y}}{\partial x}\right|_{x, y=0}$ is the quadrupole gradient evaluated on the $z$ axis. Defining $Q$ to be the integrated quadrupole field gradient,

$$
\left.Q \equiv L_{\text {quad }} \frac{\partial B_{y}}{\partial x}\right|_{x, y=0},
$$

allows us to write the focal strength more concisely as the integrated field divided by the beam's momentum:

$$
\frac{1}{f}=\frac{e Q}{\beta \gamma m c} .
$$

Let $\Sigma$ represent a $4 \times 4$ beam matrix whose elements describe an ellipse in $\left(x, x^{\prime}, y, y^{\prime}\right)$ space. The diagonal elements of the $\Sigma$ matrix are the beam size or divergence for each dimension squared. Transforming the beam matrix $\Sigma(0)$ through the rotated quadrupole gives the final beam matrix $\Sigma(1)$ :

$$
\Sigma(1)=R_{\text {rotquad }} \Sigma(0) R_{\text {rotquad }}^{T} .
$$

If we assume the initial beam is collimated with perfectly parallel rays, then the emittance is zero, and $\Sigma(0)$ is

$$
\Sigma(0) \equiv\left(\begin{array}{cccc}
\Sigma_{x x}(0) & 0 & 0 & 0 \\
0 & 0 & 0 & 0 \\
0 & 0 & \Sigma_{y y}(0) & 0 \\
0 & 0 & 0 & 0
\end{array}\right) .
$$

The nonzero beam matrix elements of $\Sigma(0)$ are equal to the horizontal and vertical beam sizes squared:

$$
\Sigma_{x x}(0)=\sigma_{x}^{2} \quad \text { and } \quad \Sigma_{y y}(0)=\sigma_{y}^{2} .
$$

The volume of the beam ellipsoid in four dimensions gives the normalized 4D-emittance:

$$
\epsilon_{n, 4 \mathrm{D}}=\beta \gamma \sqrt{\operatorname{det} \Sigma} .
$$

Clearly, there is no 4D-emittance growth for the rotated quadrupole, since the rotation transformation is symplectic [3]. However, the emittance does increase for the 2D phase space distributions in $x x^{\prime}$ and $y y^{\prime}$. The $x$-plane emittance growth is given by the $2 \times 2$ submatrix in the upper lefthand corner of the 4D beam matrix. Writing out the emittance in terms of this submatrix gives

$$
\epsilon_{n, x}=\beta \gamma \sqrt{\operatorname{det}\left|\begin{array}{cc}
\Sigma_{x x} & \Sigma_{x x^{\prime}} \\
\Sigma_{x x^{\prime}} & \Sigma_{x^{\prime} x^{\prime}}
\end{array}\right|} .
$$

Applying these relations and working through the matrix algebra leads to the normalized 2D emittance growth generated by a rotated quadrupole:

$$
\epsilon_{n, \text { rotquad }}=\beta \gamma \frac{\sigma_{x} \sigma_{y}}{f}|\sin 2 \alpha| .
$$

The $x$ - and $y$-plane emittance growths are equal, since the rotation affects both planes the same. As expected, there is no emittance growth for a normal quadrupole $(\alpha=0)$. Since the skew component of the rotated field strength is $\frac{1}{f_{\text {skew }}}=\frac{1}{f} \sin 2 \alpha$, Eq. (10) shows that the emittance growth of a rotated quadrupole is due solely to its skew component.

In terms of $Q$, the rotated quadrupole normalized emittance becomes

$$
\epsilon_{n, \text { rotquad }}=\sigma_{x} \sigma_{y} \frac{e Q}{m c}|\sin 2 \alpha| .
$$

Thus, the normalized coupled transverse emittance growth is a simple product of the beam sizes, the integrated quadrupole field, and the sine function of twice the quadrupole rotation angle.

\section{FIELDS OF THE SOLENOID}

\section{A. The ideal solenoid}

The fields of the ideal solenoid have axial symmetry about the $z$ axis in cylindrical coordinates. Therefore, the fields are independent of the azimuth angle with $B_{\theta}=0$. Radial integration of $\vec{\nabla} \cdot \vec{B}=0$ leads to the following wellknown relation for fields with axial symmetry [11]:

$$
B_{r}=-\frac{r}{2} \frac{\partial B_{z}}{\partial z} .
$$

Thus, the slope of the $B_{z}$ field, $\frac{\partial B_{z}}{\partial z}$, determines the location and extent of the solenoid's fringe fields. These radial fields give the electrons a momentum kick in the $\theta$ direction which begins the beam's rotation in the solenoid. An opposite kick at the exit (due to $B_{z}$ 's opposite slope) cancels the initial azimuthal kick, so the beam exits with zero azimuthal momentum.

Using Eq. (12) for the radial field, the focal strength of a solenoid is found to depend upon the solenoid's maximum interior field squared:

$$
\frac{1}{f_{\mathrm{sol}}}=\frac{e^{2} B_{0}^{2} L_{\mathrm{sol}}}{2(\gamma \beta m c)^{2}} .
$$

Here the maximum interior field is $B_{0}$, the effective length of the solenoid is $L_{\mathrm{sol}}$, and the beam's total momentum is $\gamma \beta m c$.

The ideal solenoid generates little emittance growth for small, low energy spread beams. However, the growth can be significant for large beams due to spherical aberrations and for beams with energy spread [12]. Computing the spherical emittance requires knowing the fields at large radii either by measurement, by analytic extrapolation, or with a magnetic field finite element code. These radial 
fields can then be either integrated numerically for the field integrals or used directly in a beam simulation code to numerically compute the emittance as a function of the initial beam size to obtain the spherical emittance growth.

\section{B. Solenoid with quadrupole fields}

Although the ideal solenoid has only radial and longitudinal fields, its field can excite the surrounding magnetic material and generate azimuthal fields. In our experience, these extraneous materials (such as a vacuum pipe with magnetic welds), which are excited by the solenoid's field, often produce the strongest multipole fields and are therefore the most likely to cause emittance growth.

Once again $\vec{\nabla} \cdot \vec{B}=0$, but now there is an extra term for the azimuthal field:

$$
\frac{1}{r} \frac{\partial}{\partial r}\left(r B_{r}\right)+\frac{1}{r} \frac{\partial B_{\theta}}{\partial \theta}+\frac{\partial B_{z}}{\partial z}=0
$$

Multiplying by $r d r$ and integrating gives the more general form of Eq. (12) which includes the azimuth field gradient:

$$
B_{r}+\frac{\partial B_{\theta}}{\partial \theta}=-\frac{r}{2} \frac{\partial B_{z}}{\partial z}
$$

Thus, the slope of the longitudinal field equals the total strength of the transverse fields. This is like the focusing by a dipole magnet, where tilting the poles increases the vertical focusing but it also lowers the horizontal focal strength. For a dipole field, the sum of the two transverse strengths equals the bend angle over the bend radius [13].

Measurements are necessary to determine both the strength and multipolarity of the $B_{\theta}$ field and if it depends upon the solenoid's field or not. If the $B_{\theta}$ field does not scale with the solenoid field, then these fields are referred to as stray quadrupole fields. Stray fields are produced by magnetic materials or devices which are located near the beam line but are not magnetically connected with the solenoid's field. However, if $B_{\theta}$ is proportional to the solenoid field, then this field is referred to as the solenoid's anomalous field. These fields are called anomalous because they are unexpected irregularities or anomalies of the solenoid's field. Anomalous quadrupole fields can be caused by quadrupolelike features in the solenoid's coil or yoke design or by magnetic material placed (unintentionally) within the solenoid's magnetic circuit. Both anomalous and stray quadrupole fields can increase the emittance if they are rotated, as just discussed in Sec. II, or if they precede a solenoid, as discussed later. The next section describes magnetic field measurements of the Linac Coherent Light Source (LCLS) gun solenoid.

\section{Multipole field measurements of a solenoid}

Figure 2 shows magnetic measurements for the LCLS-I gun solenoid [14]. The upper plot is the $z$ dependence of the longitudinal field, as measured using a three-axis Hall probe. The lower plot is the integrated quadrupole gradient and rotation angle as a function of $z$. The integrated quadrupole field gradient as measured by a short rotating coil is

$$
Q_{\text {meas }} \equiv L_{\text {coil }} \frac{B_{2}\left(r=r_{\text {coil }}\right)}{r_{\text {coil }}}
$$

Here $L_{\text {coil }}$ is the axial length of the rotating coil, $r_{\text {coil }}$ is the radius of the coil, and $B_{2}\left(r=r_{\text {coil }}\right)$ is the quadrupole field at the coil's radius.

The rotating coil measurements show that quadrupole fields peak at the ends of the solenoid as expected from the previous discussion in Sec. III B. The data also show that the quadrupole angle changes $90^{\circ}$ between the ends, which corresponds to a polarity reversal. In addition, the quadrupole fields scale with the solenoid's field. Therefore, they qualify as anomalous quadrupole fields of the solenoid. These properties suggest that there is some magnetic

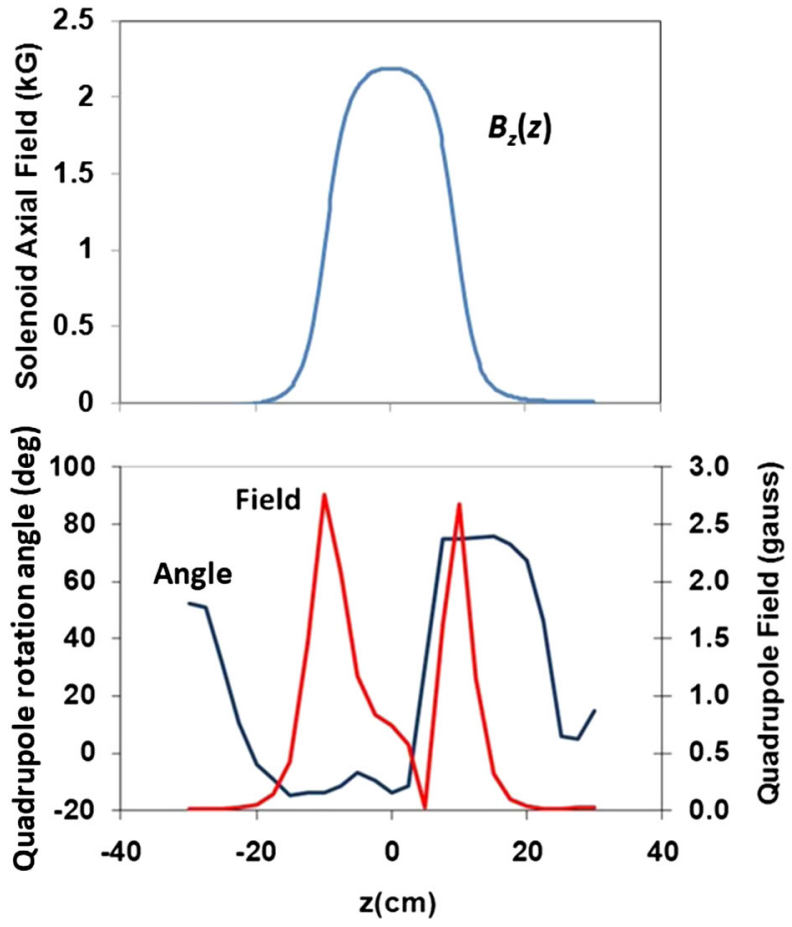

FIG. 2. Magnetic measurements of the LCLS gun solenoid for an integrated field of $0.046 \mathrm{~T}-\mathrm{m}$. Top: Hall probe measurements of the solenoid axial field. The transverse location of the measurement axis (the $z$ axis) was determined by minimizing the radial field. Bottom: Rotating coil measurements of the quadrupole field. The rotating coil dimensions were $2.5 \mathrm{~cm}$ long with a $2.8 \mathrm{~cm}$ radius. The measured quadrupole field is thus averaged over these dimensions. 
material which is unintentionally within the solenoid's magnetic field or quadrupolar coil winding error.

In our experience, the sources of these low quadrupole fields were difficult to identify and control even with stateof-the-art, finite-element-analysis calculations and following rigorous fabrication practices with a careful selection of materials. Therefore, we decided to install weak normal and skew quadrupole correctors in the LCLS-I solenoid and optimize their settings with the beam itself. Measurements of the beam emittance taken while optimizing the quadrupole correctors are described later in Sec. IV D.

\section{COUPLED TRANSVERSE DYNAMICS IN QUADRUPOLE + SOLENOID SYSTEMS}

In this section, we develop a simple yet accurate model for understanding the effects of a quadrupole and solenoid system with coupled transverse trajectories. These theoretical studies and numerical simulations confirm that the emittance growth is due to well-defined coupled dynamics between the transverse planes. In addition, the theory, simulation, and experiments show that the emittance growth can be canceled with a correcting quadrupole field.

In this theory, the full 4D transverse transport matrix conserves the $4 \mathrm{D}$ emittance, since the transformation is linear in four dimensions. However, both the 2D subspaces of $x x^{\prime}$ and $y y^{\prime}$ can gain emittance because of nonzero cross terms in the beam matrix. The linear 4D transformation generates cross terms or correlations between the $x$ and $y$ planes via nonzero off-diagonal beam matrix elements such as $\Sigma_{x x^{\prime}} \Sigma_{x y}, \Sigma_{x^{\prime} y}, \Sigma_{x^{\prime} y^{\prime}}$, etc. Since a rotated quadrupole can also create these cross terms, it is possible to use a corrector quadrupole to control them and the emittance they produce.

\section{A. Emittance due to a quadrupole field near the entrance of a solenoid}

The emittance growth of a normal quadrupole followed by a solenoid is computed assuming a normal-quadrupole field followed by a solenoid. The $\left(x, x^{\prime}, y, y^{\prime}\right)$ transformation of a beam ray through a thin quadrupole lens followed by a solenoid can be written as [15]

$$
R_{\mathrm{sol}} R_{\text {quad }}=\left(\begin{array}{cccc}
\cos ^{2} K L & \frac{\sin K L}{K} & \sin K L \cos K L & \frac{\sin ^{2} K L}{K} \\
-K \sin K L \cos K L & \cos ^{2} K L & -K \sin ^{2} K L & \sin K L \cos K L \\
-\sin K L \cos K L & -\frac{\sin ^{2} K L}{K} & \cos ^{2} K L & \frac{\sin K L \cos K L}{K} \\
K \sin ^{2} K L & -\sin K L \cos K L & -K \sin K L \cos K L & \cos ^{2} K L
\end{array}\right)\left(\begin{array}{cccc}
1 & 0 & 0 & 0 \\
-\frac{1}{f} & 1 & 0 & 0 \\
0 & 0 & 1 & 0 \\
0 & 0 & +\frac{1}{f} & 1
\end{array}\right) .
$$

Here $L$ is the effective length of the solenoid, $K \equiv \frac{e B_{0}}{2 \beta \gamma m c}$, $B_{0}$ is the maximum interior magnetic field of the solenoid, and $f$ is the focal length of the quadrupole field before the solenoid. The beam is rotated through the angle $K L$ by the solenoid.

As shown earlier, an initial $4 \times 4$ beam matrix $\Sigma(0)$ can be transported through the quadrupole and solenoid producing the exit beam matrix $\Sigma(1)$ :

$$
\Sigma(1)=R_{\mathrm{sol}} R_{\text {quad }} \Sigma(0)\left(R_{\mathrm{sol}} R_{\text {quad }}\right)^{T} .
$$

Using the same $\Sigma(0)$ for a perfectly parallel beam as before and working through tedious matrix algebra gives the expected result for the transverse-plane emittance growth of a normal quadrupole followed by a solenoid as

$$
\epsilon_{n, \mathrm{quad}+\mathrm{sol}}=\beta \gamma \frac{\sigma_{x, \text { sol }} \sigma_{y, \text { sol }}}{f}|\sin 2 K L| .
$$

In other words, the emittance growth of a normal quadrupole followed by a solenoid is that of the quadrupole rotated the Larmor angle of the solenoid. The emittance growth is the same for both the $x$ and $y$ planes.

Substituting the integrated quadrupole field for $1 / f$, one finds the coupled transverse dynamics normalized emittance growth depending only upon the beam size, the integrated quadrupole field, and the rotation angle of the beam in the solenoid:

$$
\epsilon_{n, \mathrm{quad}+\mathrm{sol}}=\sigma_{x, \mathrm{sol}} \sigma_{y, \mathrm{sol}} \frac{e Q}{m c}|\sin 2 K L| .
$$

Figure 3 compares this simple formula with a particle tracking simulation for a solenoid preceded by a normalquadrupole field. The initial beam had zero emittance and zero energy spread and was circular and uniform. No spacecharge forces are included in the simulation. The figure shows the normalized emittances given by Eq. (19) and the simulation, plotted as a function of the rms beam size at the solenoid entrance. The normal-quadrupole focal length is $50 \mathrm{~m}$ for a $6 \mathrm{MeV}$ beam energy. This corresponds to an integrated quadrupole field gradient of $4.3 \mathrm{G}$, which is similar to the measured field of the LCLS solenoid (see Fig. 2). The analytic theory and the simulation assume a short quadrupole field with this integrated quadrupole field located at the solenoid's entrance. The simulation emittance is slightly larger, since it includes both the coupled transverse dynamics emittance being discussed here and the geometric aberration. The good agreement verifies the model's assumptions and illustrates that even a weak, 


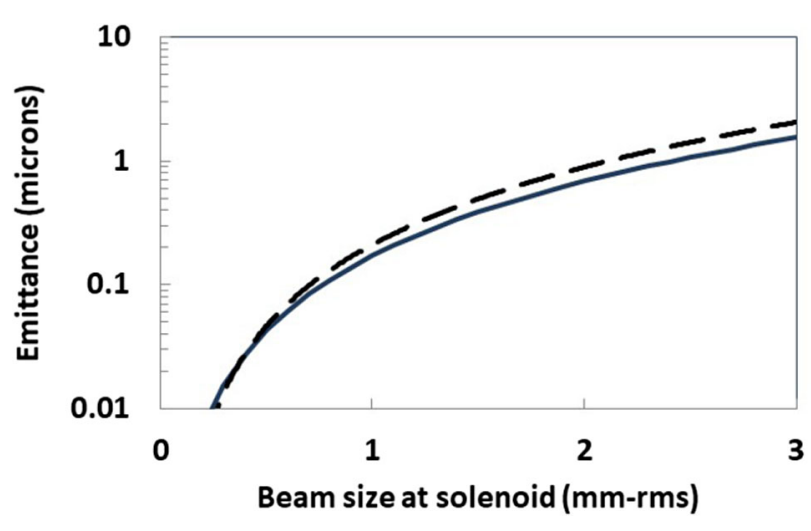

FIG. 3. Normalized emittance due to the quadrupole-solenoid coupling given by Eq. (19) (solid line) and by a particle tracking simulation (dashed line) for the case of the LCLS solenoid. For a beam energy of $6 \mathrm{MeV}$, the quadrupole focal length was $50 \mathrm{~m}$. This corresponds to an integrated quadrupole field of $4.3 \mathrm{G}$. The solenoid had an integrated field of $0.046 \mathrm{~T}-\mathrm{m}$, giving a focal length of $\sim 12 \mathrm{~cm}$. The simulation is done with the GPT code [16].

normal-quadrupole field can produce significant emittance growth when combined with the rotation in a solenoid field.

If the quadrupole field is rotated an angle $\alpha$, as shown in Fig. 1, then, by induction, one adds the quadrupole's rotation angle with the solenoid rotation angle to obtain the emittance:

$$
\epsilon_{n, \mathrm{quad}+\mathrm{sol}}(\alpha)=\sigma_{x, \mathrm{sol}} \sigma_{y, \mathrm{sol}} \frac{e Q}{m c}|\sin 2(K L+\alpha)| .
$$

In Fig. 4, the emittance growth given by Eq. (21) and simulations is plotted as a function of the quadrupole angle of rotation. The integrated quadrupole field is $Q=4.3 \mathrm{G}$, followed by a strong solenoid (focal length of $12 \mathrm{~cm}$, integrated field of $0.046 \mathrm{~T} \mathrm{~m}$ ). The beam energy used in the simulation was $6 \mathrm{MeV}$. At this beam energy, a $4.3 \mathrm{G}$ quadrupole field focuses the beam with a 50-m focal length. In both the analytic theory and the simulation, the emittance growth becomes zero whenever

$$
K L+\alpha=\frac{\pi}{2}
$$

or every $90^{\circ}$. The slight offset in the emittance and angle between the theory and simulation could result from the third-order emittance of the solenoid's nonlinear radial fringe fields which are included in the simulation but not in the model.

\section{B. Cancellation of the emittance due to transverse coupling in a solenoid}

In this subsection, the solenoid and quadrupole fields are modeled for a quadrupole + solenoid + quadrupole (qsq) configuration. Figure 5 shows the model layout and the quadrupole and solenoid parameters used to compare the analytic theory with numerical simulations. The quadrupole corrector, or quad corrector, used to cancel the transverse coupled emittance is located after the solenoid.

If the solenoid is a thick lens, then the beam size will be smaller at the exit than the entrance. However, the previous discussion showed that the coupled dynamics emittance depends upon the square of the beam size. Therefore, it is important to use different beam sizes for the solenoid and quadrupole and the quad corrector. For the case considered here, simulations give the beam size at the corrector as $0.55 \mathrm{~mm} \mathrm{rms}$ when the beam is $1 \mathrm{~mm} \mathrm{rms}$ at the solenoid entrance.

This effect is easily added to the theoretical model by defining the $x$ - and $y$-rms beam sizes at the quadrupole as $\sigma_{x, \text { quad }}$ and $\sigma_{y, \text { quad }}$, respectively, and revising the expression for the coupled transverse emittance gives

$$
\epsilon_{n, \mathrm{qsq}}=\beta \gamma\left|\frac{\sigma_{x, \mathrm{sol}} \sigma_{y, \mathrm{sol}}}{f_{1}} \sin 2\left(K L+\alpha_{1}\right)+\frac{\sigma_{x, \mathrm{cor}} \sigma_{y, \mathrm{cor}}}{f_{\mathrm{cor}}} \sin 2 \alpha_{\mathrm{cor}}\right| .
$$

The focal length for the rotated-quadrupole field preceding the solenoid is $f_{1}$, and its rotation angle is $\alpha_{1}$. The
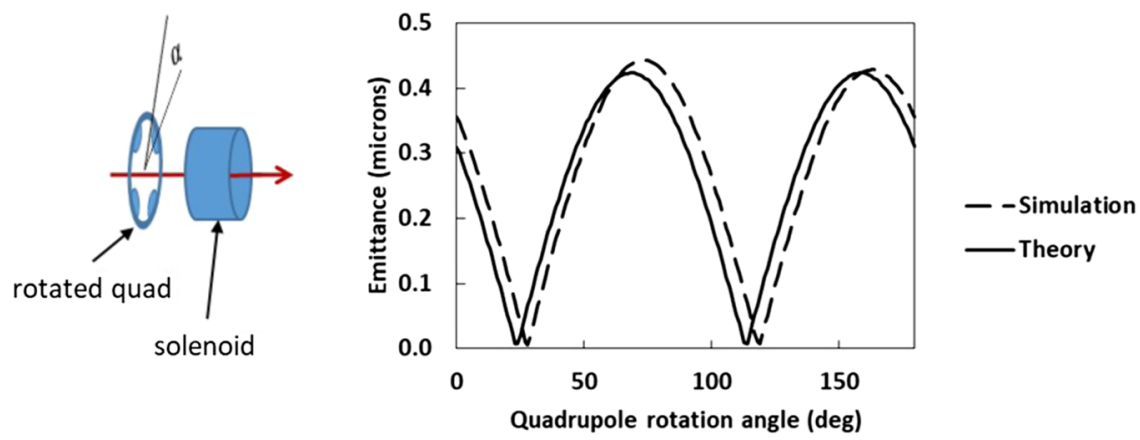

FIG. 4. The emittance growth for a quadrupole-solenoid system (shown on the left) as a function of the quadrupole rotation angle. The theory (solid line) is computed using Eq. (21) and the simulation (dashed line) is done with the GPT code. The initial beam size at the solenoid is $1 \mathrm{~mm}-\mathrm{rms}$ for both the $x$ and $y$ planes. The quadrupole and solenoid fields are the same as Fig. 3. 
Solenoid:

$\mathrm{KL}=1.16$, Eff. Length $=0.1935 \mathrm{~m}$

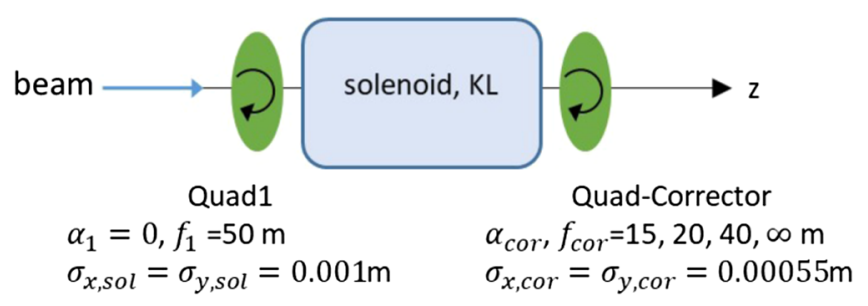

FIG. 5. Quadrupole-solenoid-quadrupole configuration and parameters used to compare emittance growth computed with the analytic model and numerical simulations shown in Fig. 6. The beam energy is $6 \mathrm{MeV}$.

quadrupole after the solenoid is a quad corrector with focal length $f_{\text {cor }}$ and rotation angle $\alpha_{\text {cor }}$.

The layout and parameters for this $\mathrm{q}_{1} \mathrm{~s}_{\mathrm{ol}} \mathrm{q}_{\mathrm{cor}}$ system given in Fig. 5 are used to compare Eq. (23) with numerical simulations. As shown in the figure, the beam size at the quad corrector is approximately half its size at quad1, as determined from the simulation.

Figure 6 shows normalized emittance growths of the analytic theory and numerical simulation for the $\mathrm{q}_{1} \mathrm{~s}_{\mathrm{ol}} \mathrm{q}_{\text {cor }}$ configuration with the parameters given in Fig. 5. The plots show the normalized $x$-plane emittance for a $6 \mathrm{MeV}$ beam as a function of the quad-corrector rotation for the theory (left) and simulation (right). In all cases, quad1 is a normal quadrupole with a $50-\mathrm{m}$ focal length. The emittance is plotted for quad-corrector focal lengths of 15, 20, 40, and infinite meters. The 50-m focal length corresponds to an integrated quadrupole gradient of $4.3 \mathrm{G}$, and the 15-, 20-, and 40-m focal lengths correspond to $Q$ values of 21.5 , 14.3, and $5.4 \mathrm{G}$, respectively.

It is important to point out that the quad corrector can be located almost anywhere, even before the solenoid. This is because the quadrupole fields have very long focal lengths and, within the thin lens approximation, the spacing between and location of the elements becomes irrelevant. Therefore, the focal strengths simply add. However, since the quad-corrector focal strength scales with the inverse beam size squared, it should be located where the beam is large. This will minimize the quad-corrector strength needed to cancel the anomalous quadrupole fields.

\section{Simulation and measurements of an injector with a quadrupole field in a solenoid}

The effectiveness of the quadrupole correction has been validated with the simulations of the LCLS injector. The LCLS injector includes 5.5-MeV rf gun, a main solenoid for emittance compensation, and two linac sections to boost energy to $135 \mathrm{MeV}$ [17]. The ASTRA code [18] including 3D space-charge forces is used to simulation the LCLS injector. Figure 7 shows the emittance vs $z$ for no quadrupole fields with only the solenoid field (blue line), the emittance due to a weak normal quadrupole (focal length $11.5 \mathrm{~m}$ ) at the entrance of the solenoid (green line), and the weak quadrupole emittance corrected by a quadrupole with a focal length of $28.6 \mathrm{~m}$ and rotation angle of $11.5^{\circ}$ after the solenoid (red line). These results show that the emittance grows about $20 \%$ if there is a weak quadrupole field situated at the entrance of the solenoid. The red curve shows this emittance growth can be completely corrected by a weak, rotated quadrupole in the region downstream of the solenoid. Therefore, the simulations verify the analyses of the previous sections.

Two long quadrupole correctors, one normal and one skew, are installed inside of the solenoid for the LCLS-I injector [14]. Figure 8 shows one example of the experimental data of the injector emittance measured at $135 \mathrm{MeV}$ vs the skew quadrupole corrector field strength. Shown are typical measurements made during LCLS-I operations to optimize the beam emittance.
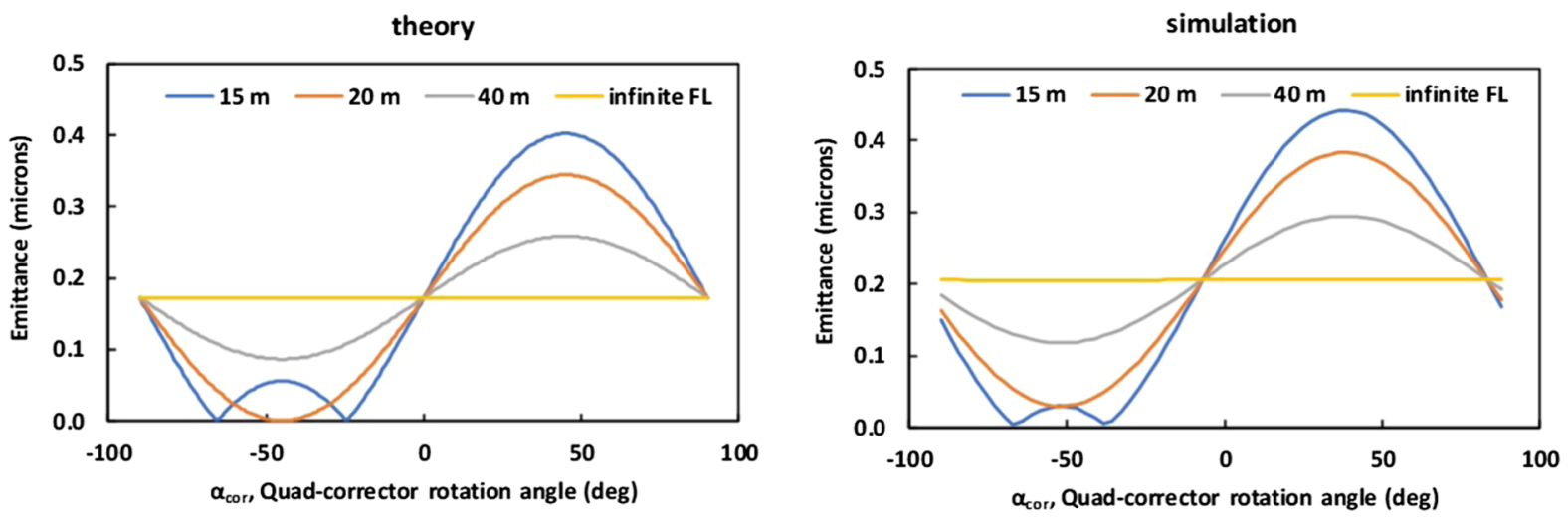

FIG. 6. Comparison of the transverse coupled emittance for a $\mathrm{q}_{1} \mathrm{~s}_{\mathrm{ol}} \mathrm{q}_{\mathrm{cor}}$ configuration as a function of the quad-corrector rotation angle as computed by Eq. (23) (left) and a numerical simulation (right). The configuration and parameters are given in Fig. 5 . The emittance growth is for quad1 with a fixed focal length of $50 \mathrm{~m}$ and quad-corrector focal lengths of 15, 20, 40, and infinite meters. The beam energy is $6 \mathrm{MeV}$. The 15-, 20-, and 40-m focal lengths correspond to 21.5, 14.3, and 5.4 G, respectively, integrated fields. 


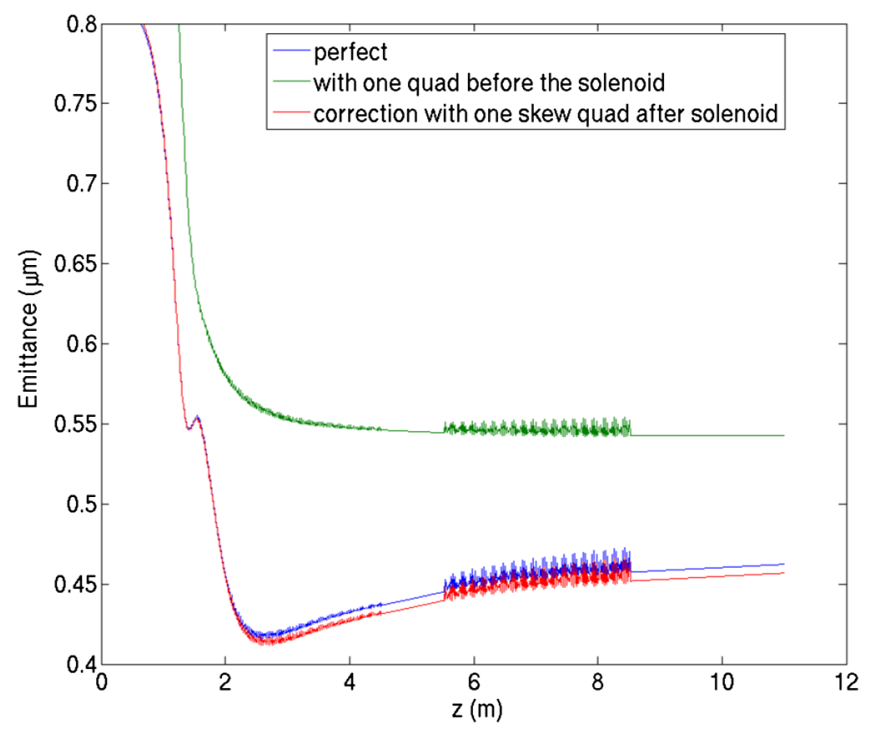

FIG. 7. Simulations of emittance cancellation with a quadrupole corrector: The emittance without a quadrupole field at the solenoid entrance (blue line), the emittance with a normal quadrupole at the entrance of the solenoid (green line), and emittance correction with a quadrupole corrector downstream of the solenoid (red line).

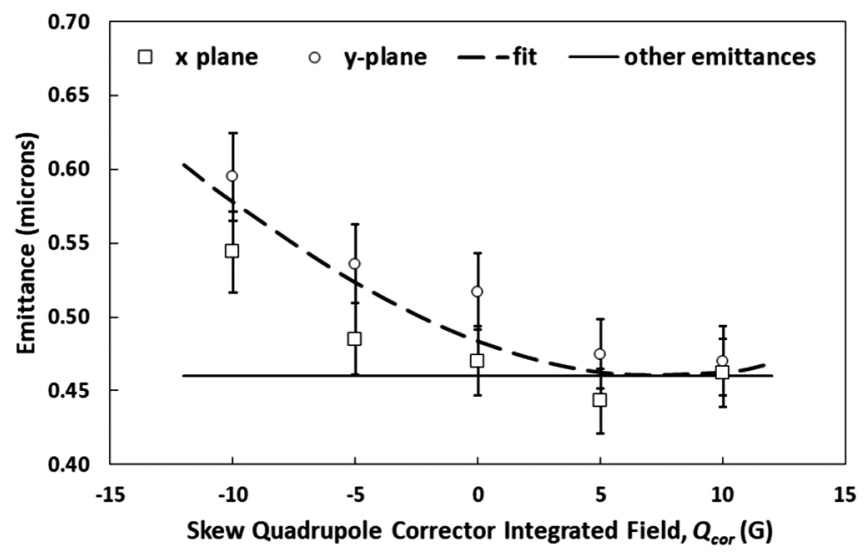

FIG. 8. LCLS injector emittance at $100 \mathrm{pC}$ for the $x$ and $y$ planes vs the skew quadrupole corrector integrated field strength $Q_{\text {cor }}$. The emittance (points w/error bars) was measured at a beam energy of $135 \mathrm{MeV}$. The dashed curve shows the fit to the data with Eq. (24). The other-effects emittance $\epsilon_{\text {other }}$ is shown by the solid line at $0.46 \mu \mathrm{m}$. The fit indicates that the coupled transverse dynamics emittance growth $\epsilon_{\text {coupled }}$ is $0.15 \mu \mathrm{m}$.

The data in Fig. 8 have been fit with the quadratic sum of the quadrupole-solenoid-quadrupole emittance with the other unknown but constant emittances $\epsilon_{\text {other }}$, using the following expression:

$$
\epsilon_{\text {expt }}=\sqrt{\epsilon_{\text {other }}^{2}+\left(\epsilon_{\text {coupled }}+\frac{e}{m c} \sigma_{x, \text { cor }} \sigma_{y, \text { cor }} Q_{\text {cor }}\right)^{2}} .
$$

Here $\epsilon_{\text {coupled }}$ is the emittance growth due to the uncorrected coupled transverse dynamics of the injector, and $Q_{\text {cor }}$ is the integrated quadrupole field gradient of the quadrupole corrector. The $x$-rms and $y$-rms beam sizes at the quad corrector are $\sigma_{x, \text { cor }}$ and $\sigma_{y, \text { cor }}$, respectively. The fit to the data assumes that these are the same and equal to $0.6 \mathrm{~mm}$ rms. Since the data were taken using the skew quadrupole corrector, the $\sin 2 \alpha$ factor in Eq. (23) is equal to one. A single curve has been fit to both the $x$ - and $y$-plane emittances. However, the offset in emittance between the $x$ and $y$ planes suggests that the other-effects emittance for the $y$ plane is approximately $25 \mathrm{~nm}$ larger than the $x$ plane's other emittance. The data are shown with 5\% error bars.

The fit (dashed line) shows that the emittance is minimized when the integrated field of the skew quad corrector is $7 \mathrm{G}$, corresponding to a 31-m focal length for a $6 \mathrm{MeV}$ beam. The emittance due to other effects is $\epsilon_{\text {other }}=$ $0.46 \mu \mathrm{m}$ (solid line). The fit is the quadrature sum of this emittance and the total coupled transverse dynamics emittance growth given inside the second set of brackets of Eq. (24). The total coupled transverse dynamics emittance growth is the sum of the unwanted coupled emittance, $\epsilon_{\text {coupled }}$, and the quad corrector's emittance. Since the quadcorrector field can be positive or negative, it can have the opposite sign of the unwanted coupled emittance and cancel it. The fit gives $\epsilon_{\text {coupled }}=0.15 \mu \mathrm{m}$ for the coupled transverse dynamics emittance growth. Since the rf cavities of this injector were designed to have compensated rf quadrupole fields [14], this emittance is most likely due to the anomalous quadrupole fields of the solenoid. These results are consistent with the measured fields shown in Fig. 2.

\section{COUPLED TRANSVERSE DYNAMICS DUE TO THE ROTATED FIELDS OF RF COUPLERS}

This section discusses the quadrupole fields of an $\mathrm{rf}$ coupler. These fields are linear in $x$ and $y$ near the beam axis but rotated about the beam axis due to the mechanical constraints of the coupler design. It is shown by calculation and simulation that the emittance generated by these coupler fields can be cancelled with a dc corrector quadrupole field.

\section{A. Analysis of the rf coupler field}

Maxwell's equations show that the transverse electric fields over a small region near the beam axis can be specified as a linear expansion obeying the following relations between the field gradients [19]:

$$
\begin{aligned}
& E_{x}=E_{x, 0}+\frac{\partial E_{x}}{\partial x} x+\frac{\partial E_{x}}{\partial y} y \\
& E_{y}=E_{y, 0}+\frac{\partial E_{x}}{\partial y} x-\frac{\partial E_{x}}{\partial x} y
\end{aligned}
$$

A coupler gives the beam an instantaneous kick in voltage along the $x, y$, and $z$ directions. Each component 
of the kick gives the beam an instantaneous jump in voltage when the beam transits the coupler. Following the literature [20], the complex voltage kick factor is defined as

$$
\vec{v}(x, y) \equiv \frac{\vec{V}(x, y)}{V_{z}(0,0)} \cong\left(\begin{array}{c}
v_{x 0}+v_{x x} x+v_{x y} y \\
v_{y 0}+v_{y x} x+v_{y y} y \\
1+\cdots
\end{array}\right)
$$

where the complex voltage kick $\vec{V}(x, y)$ is given by integrals of the coupler fields along lines parallel to the $z$ axis (beam's optical axis):

$$
\vec{V}(x, y)=\int[\vec{E}(\vec{r})+i c \vec{\beta} \times \vec{B}(\vec{r})] e^{i \omega z / c} d z
$$

The $\vec{B}$ term is imaginary to account for its $\pi / 2 \mathrm{rf}$ phase shift in time with respect to the electric field. The complex voltage kick factor gives the electrons a momentum impulse of [20]

$$
\vec{p}=\operatorname{Re}\left\{\vec{v}(x, y) e^{i \phi_{s}}\right\} \frac{e V_{\mathrm{acc}}}{c} .
$$

Here $\omega$ is the rf frequency and $c$ is the speed of light. Equation (29) is the transverse momentum of an electron at a distance $s$ behind the head electron and having phase $\phi_{s}=\frac{\omega s}{c}+\phi_{\text {head }}$ with respect to the coupler's rf waveform. This phase relation assumes that the beam is relativistic. Writing out the components of the coupler's $x-y$ plane momentum kick shows that the spatial and phase dependences can be separated into a complex voltage amplitude and phase, whose real part gives the transverse momentum kick of the coupler:

$$
\left(\begin{array}{c}
p_{x} \\
p_{y}
\end{array}\right)_{\text {coupler }}=\frac{e V_{\text {acc }}}{c} \operatorname{Re}\left\{\left(\begin{array}{c}
v_{0 x}+v_{x x} x+v_{x y} y \\
v_{0 y}+v_{y x} x+v_{y y} y
\end{array}\right) e^{i \phi_{s}}\right\} .
$$

Dividing by the total momentum converts this to the coupler's angle kick vector:

$$
\left(\begin{array}{c}
x^{\prime} \\
y^{\prime}
\end{array}\right)_{\text {coupler }}=\frac{e V_{\mathrm{acc}}}{\beta \gamma m c^{2}} \operatorname{Re}\left\{\left(\begin{array}{c}
v_{0 x}+v_{x x} x+v_{x y} y \\
v_{0 y}+v_{y x} x+v_{y y} y
\end{array}\right) e^{i \phi_{s}}\right\} .
$$

Applying the relation between the field gradients shown in Eqs. (25) and (26) reduces the number of independent elements of the kick matrix from four to two:

$$
\left(\begin{array}{c}
x^{\prime} \\
y^{\prime}
\end{array}\right)_{\text {coupler }}=\frac{e V_{\text {acc }}}{\beta \gamma m c^{2}} \operatorname{Re}\left\{\left(\begin{array}{c}
v_{0 x}+v_{x x} x+v_{x y} y \\
v_{0 y}+v_{x y} x-v_{x x} y
\end{array}\right) e^{i \phi_{s}}\right\} .
$$

Absorbing the various factors and phase (time) dependence into a renormalized voltage kick matrix given by $\tilde{v}$ allows one to write

$$
\left(\begin{array}{c}
x^{\prime} \\
y^{\prime}
\end{array}\right)_{\text {coupler }}=\left(\begin{array}{l}
\tilde{v}_{0 x}+\tilde{v}_{x x} x+\tilde{v}_{x y} y \\
\tilde{v}_{0 y}+\tilde{v}_{x y} x-\tilde{v}_{x x} y
\end{array}\right) .
$$

The $\tilde{v}_{0 x, 0 y}$ terms are the coupler's dipole kicks which can be canceled with nearby steering dipoles. Simulations show that dipole steering is very effective at mitigating the dipolekick emittance produced when the beam goes through the coupler at the wrong angle and/or transverse position.

The $\tilde{v}_{x x}$ terms indicate uncoupled $x$-plane focusing, because the final angle kick is proportional to $x$, and similarly for the $y$ plane. Thus, $\tilde{v}_{x x}$ is the focal strength of the normal-quadrupole component of the rf field. This focusing does not produce any emittance growth, except that due to the time dependence of the rf fields. The cross term $\tilde{v}_{x y}$ is related to the skew component of the rf's quadrupole field and does generate emittance growth. As will be shown later, this is similar to the growth generated by the beam's rotation in a solenoid and can be canceled using a rotated quadrupole.

\section{B. The emittance due to rotated rf-coupler quadrupole fields}

For simplicity, let us assume that the electron bunch distribution in the $x y$ plane is uniform inside a square area having dimensions $-R<x<R$ by $-R<y<R$. This trivial distribution simplifies the calculation of the variances and averages needed for deriving the coupler-induced emittance while retaining the essential physics. The variance and standard deviation for this $2 R \times 2 R$ square uniform distribution are, respectively,

$$
\left\langle x^{2}\right\rangle=\left\langle y^{2}\right\rangle=\frac{R^{2}}{3} \quad \text { and } \quad \sigma_{x}=\frac{R}{\sqrt{3}} .
$$

The normalized emittance for the $x$ plane is defined as

$$
\epsilon_{n}=\beta \gamma \sqrt{\left\langle x^{2}\right\rangle\left\langle x^{\prime 2}\right\rangle-\left\langle x x^{\prime 2}\right\rangle}
$$

Here it is important to include the correlation $\left\langle x x^{\prime}\right\rangle$ term in the emittance definition. After some tedious algebra, the coupler-induced emittance is found to be solely due to the cross term $v_{x y}$ of the complex voltage kick: 


$$
\begin{aligned}
\epsilon_{n, \text { coupler }}(s)= & \frac{e V_{\text {acc }}}{m c^{2}} \sigma_{x}^{2} \mid v_{x y}^{r} \cos \left(\frac{\omega s}{c}+\phi_{\text {head }}\right) \\
& +v_{x y}^{i} \sin \left(\frac{\omega s}{c}+\phi_{\text {head }}\right) \mid
\end{aligned}
$$

Here $v_{x y}^{r}$ and $v_{x y}^{i}$ are the real and imaginary parts, respectively, of $v_{x y}$.

Equation (36) gives the transverse emittance of a thin slice of the bunch, a distance $s$ behind the bunch head. The rf phase of the bunch head is $\phi_{\text {head }}$, and the tail is a bunch length, $l_{\text {bunch }}$, behind it at the rf phase of $\frac{\omega l_{\text {bunch }}}{c}+\phi_{\text {head }}$. Figure 9 shows as an example the head and tail emittances vs the rf phase for a head-tail phase difference of 10 degrf. (Here the unit degrf is defined as one degree of phase at the $\mathrm{rf}$ frequency of interest, which, in this case, is 1.3 GHz.) The head minus the tail emittance is also plotted and shows that the difference is $20 \mathrm{~nm}$ or less, which is small compared to the uncorrected emittance of more than $100 \mathrm{~nm}$; confirming the effect is mostly due to the skewed quadrupole field rather than the phasedependent kick.

The emittance over the length of the bunch can be computed by averaging the slice emittance in Eq. (36) over the longitudinal distribution of electrons. Assuming the longitudinal distribution is uniform with full width, $l_{\text {bunch }}$, then the bunch average emittance can be found from

$$
\left\langle\epsilon_{n, \text { coupler }}\right\rangle=\frac{\int_{0}^{l_{\text {bunch }}} \epsilon_{n, \text { coupler }}(s) d s}{\int_{0}^{l_{\text {bunch }}} d s} .
$$

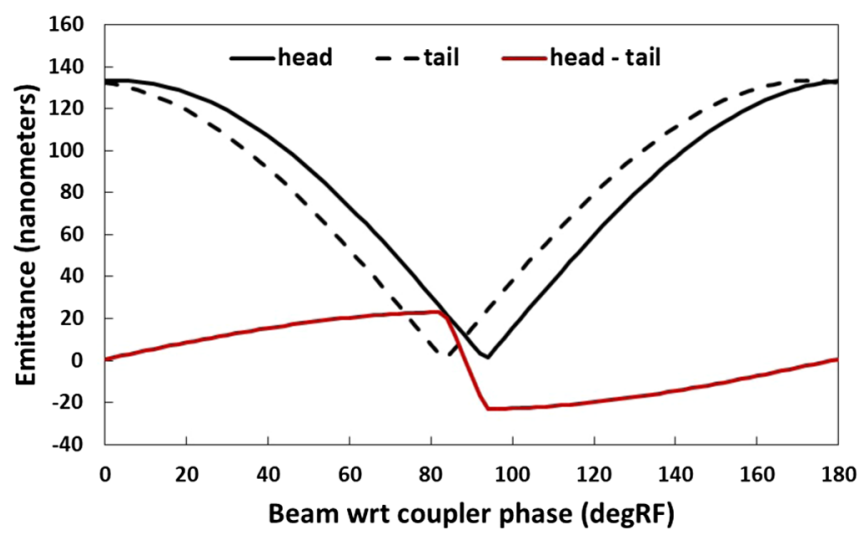

FIG. 9. The head ( $s=0$, solid line) and tail $\left(\frac{\omega s_{\text {tail }}}{c}=10\right.$ degrf, dashed line) emittances vs phase for $\sigma_{x}=1 \mathrm{~mm}$. The emittance difference in the head and tail (red line) is a maximum when the beam is on the crest of the rf waveform. The coupler voltage and kick are $V_{\text {acc }}=20 \mathrm{MV}$ and $v_{x y}=(3.4+0.2 i) \times 10^{-6} / \mathrm{mm}$, respectively, which are typical parameters for SRF cavities [20]. Here the unit degrf is a degree of phase at the rf frequency of $1.3 \mathrm{GHz}$.
Inserting the coupler slice emittance gives

$$
\begin{aligned}
\left\langle\epsilon_{n, \text { coupler }}\right\rangle= & \frac{e V_{\text {acc }}}{m c^{2}} \sigma_{x}^{2} \mid v_{x y}^{r}\left\langle\cos \left(\frac{\omega s}{c}+\phi_{\text {head }}\right)\right\rangle \\
& +v_{x y}^{i}\left\langle\sin \left(\frac{\omega s}{c}+\phi_{\text {head }}\right)\right\rangle \mid
\end{aligned}
$$

Taking the averages and expanding in terms of the bunch length gives the projected emittance of the bunch:

$$
\begin{aligned}
\left\langle\epsilon_{n, \text { coupler }}\right\rangle= & \frac{e V_{\text {acc }}}{m c^{2}} \sigma_{x}^{2} \mid\left(v_{x y}^{r} \cos \phi_{\text {head }}+v_{x y}^{i} \sin \phi_{\text {head }}\right) \\
& -\left(v_{x y}^{r} \sin \phi_{\text {head }}+v_{x y}^{i} \cos \phi_{\text {head }}\right) \frac{\Delta \phi_{\text {bunch }}}{2} \mid .
\end{aligned}
$$

The first term inside the absolute value function gives the emittance growth due to the rotated transverse quadrupole field and generates emittance even for infinitesimal bunch length. The second term depends linearly upon the bunch length as well as the coupler's skew field. For the short bunches considered here $\Delta \phi_{\text {bunch }} \ll 1$, and the second term can be ignored, and the rf-coupler emittance growth becomes

$\left\langle\epsilon_{n, \text { coupler }}\right\rangle=\frac{e V_{\text {acc }}}{m c^{2}} \sigma_{x}^{2}\left|v_{x y}^{r} \cos \phi_{\text {head }}+v_{x y}^{i} \sin \phi_{\text {head }}\right|$.

It is important to note that, even if $v_{x y}=0$, there remains emittance growth from the normal-quadrupole term $v_{x x}$, due the bunch's phase length. The phase emittance occurs because the rf field is time dependent. This changing field then gives different quadrupole kicks along the bunch length and generates projected emittance growth. The first term in Eq. (39) is absent for a normal-quadrupole rf field, since a normal-quadrupole has no emittance growth. However, the bunch length dependent term remains with $v_{x y}$ replaced by $v_{x x}$. This emittance growth due to bunch length can be mitigated by shaping the rf cavity [21] or introducing additional penetrations into the cavity walls [22] to cancel both normal and skew components of the quadrupole rf field on the beam axis.

\section{Cancellation of coupler kicks with a rotated quadrupole field}

As shown in Sec. II, the kick angle vector in the $x y$ plane for a quadrupole with focal length $f$ and rotation angle $\alpha$ can be written as

$$
\left(\begin{array}{l}
x^{\prime} \\
y^{\prime}
\end{array}\right)_{\text {rotquad }}=\left(\begin{array}{c}
-\frac{\cos 2 \alpha}{f} x-\frac{\sin 2 \alpha}{f} y \\
-\frac{\sin 2 \alpha}{f} x+\frac{\cos 2 \alpha}{f} y
\end{array}\right) .
$$

Comparing Eqs. (33) and (41), one can define the normal and skew components of the coupler's quadrupole field: 
$\tilde{v}_{x x, \text { coupler }}=-\frac{\cos 2 \alpha_{\text {coupler }}}{f_{\text {coupler }}}$ and $\quad \tilde{v}_{x y, \text { coupler }}=\frac{\sin 2 \alpha_{\text {coupler }}}{f_{\text {coupler }}}$.

Here the subscript "coupler" has been added to denote that these are kicks due to the rotated quadrupole field of a rf coupler. These relations allow us to model the coupler fields equivalently as a quadrupole with focal length $f_{\text {coupler }}$ and rotated $\alpha_{\text {coupler }}$ about the $z$ axis. The rotation angle of the coupler's quadrupole field in terms of the normalized voltage kicks is

$$
\alpha_{\text {coupler }}=-\frac{1}{2} \tan ^{-1} \frac{\tilde{v}_{x y}}{\tilde{v}_{x x}} .
$$

Since the quadrupole fields are weak, we can again apply the thin lens approximation and add Eqs. (33) and (41) to give the total kick angle of the coupler and a quad corrector located near the coupler:

$$
\left(\begin{array}{c}
x^{\prime} \\
y^{\prime}
\end{array}\right)_{\text {total }}=\left(\begin{array}{c}
x^{\prime} \\
y^{\prime}
\end{array}\right)_{\text {coupler }}+\left(\begin{array}{c}
x^{\prime} \\
y^{\prime}
\end{array}\right)_{\text {quad }}=\left(\begin{array}{c}
\left\{\tilde{v}_{x x}-\frac{\cos 2 \alpha_{\text {cor }}}{f_{\text {cor }}}\right\} x+\left\{\tilde{v}_{x y}-\frac{\sin 2 \alpha_{\mathrm{cor}}}{f_{\text {cor }}}\right\} y \\
\left\{\tilde{v}_{x y}-\frac{\sin 2 \alpha_{\text {cor }}}{f_{\text {cor }}}\right\} x-\left\{\tilde{v}_{x x}-\frac{\cos 2 \alpha_{\mathrm{cor}}}{f_{\mathrm{cor}}}\right\} y
\end{array}\right) .
$$

The symmetry of Maxwell's equations, mentioned earlier in this section, can now be appreciated. Equation (44) proves that the emittance and the focusing effects of the coupler can be exactly canceled with a rotated dc quadrupole corrector. It shows that the following two equations determine the quad-corrector focal strength and rotation angle which cancel the coupler quadrupole kick:

$$
\tilde{v}_{x x}-\frac{\cos 2 \alpha_{\text {cor }}}{f_{\text {cor }}}=0 \quad \text { and } \quad \tilde{v}_{x y}-\frac{\sin 2 \alpha_{\text {cor }}}{f_{\text {cor }}}=0 .
$$

Simultaneously solving these two equations gives paired values for the quad corrector's rotation angle and focal strength which cancel the coupler field's cross term (emittance) and the quadrupole-focus term (astigmatism) for a single slice of the bunch. Solutions for the quadcorrector rotation angle and focal strength are, respectively,

$$
\begin{gathered}
\alpha_{\mathrm{cor}}=\frac{1}{2} \tan ^{-1} \frac{\tilde{v}_{x y}}{\tilde{v}_{x x}}, \\
\frac{1}{f_{\text {cor }}}=\frac{e V_{\mathrm{acc}}}{\beta \gamma m c^{2}} \sqrt{\tilde{v}_{x x}^{2}+\tilde{v}_{x y}^{2}} .
\end{gathered}
$$

and

$$
\tilde{v}_{x y}\left(\phi_{s}\right)=\frac{e V_{\mathrm{acc}}}{\beta \gamma m c^{2}}\left(v_{x y}^{r} \cos \phi_{s}-v_{x y}^{i} \sin \phi_{s}\right) .
$$

Inserting these relations into Eqs. (46) and (47) gives the quad-corrector rotation angle

$$
\alpha_{\mathrm{cor}}\left(\phi_{s}\right)=\frac{1}{2} \tan ^{-1} \frac{v_{x y}^{r} \cos \phi_{s}-v_{x y}^{i} \sin \phi_{s}}{v_{x x}^{r} \cos \phi_{s}-v_{x x}^{i} \sin \phi_{s}}
$$

$$
\frac{1}{f_{\operatorname{cor}\left(\phi_{s}\right)}}=\frac{e V_{\mathrm{acc}}}{\beta \gamma m c^{2}} \sqrt{\left(v_{x x}^{r} \cos \phi_{s}-v_{x x}^{i} \sin \phi_{s}\right)^{2}+\left(v_{x y}^{r} \cos \phi_{s}-v_{x y}^{i} \sin \phi_{s}\right)^{2}} .
$$

These solutions are for a thin slice of the bunch transiting the coupler at a bunch-rf phase of $\phi_{s}$.

As a numerical example, we use the SRF coupler parameters given in Dohlus' paper [20] to compute the corrector quadrupole requirements. His Table 1 gives the normalized complex voltage kick factors as

$$
\begin{gathered}
v_{x x}=(1-0.7 i) \times 10^{-6} / \mathrm{mm}, \\
v_{x y}=(3.4-0.2 i) \times 10^{-6} / \mathrm{mm} .
\end{gathered}
$$

These coupler kicks correspond to a normalizing voltage of $20 \mathrm{MV}$ and assume a beam kinetic energy of $800 \mathrm{keV}$ 


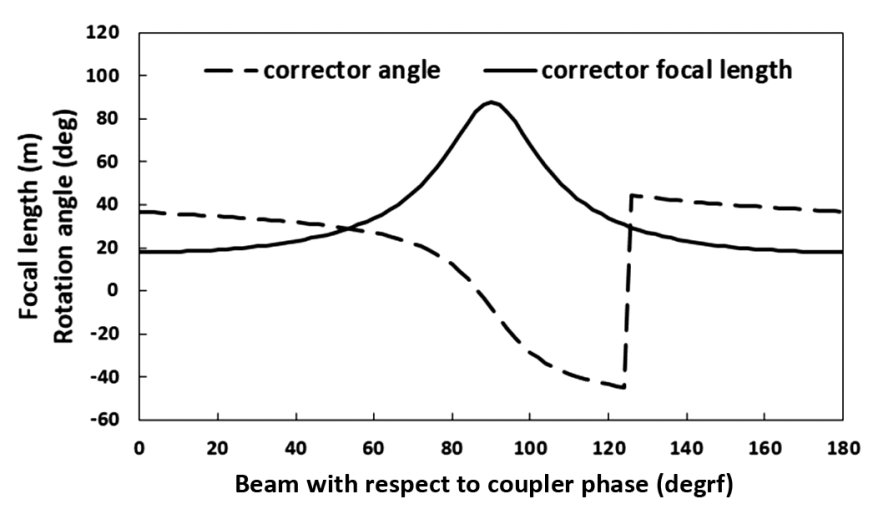

FIG. 10. The correction quadrupole rotation angle (dashed line) and focal length (solid line) vs the beam to coupler rf phase for a high-power coupler of a SRF linac. The corrector rotation angle is discontinuous near the coupler phase of $\sim 125$ degrf where the denominator of Eq. (50) is zero and the $\frac{1}{2} \tan ^{-1}$ function jumps to stay within its principal value range of $-45^{\circ}$ and $45^{\circ}$.

such that $\beta \gamma=2.5$. Figure 10 shows the focal length and rotation angle required to correct for these complex voltage kicks as functions of the beam-to-rf phase. The correction quadrupole rotation angle and focal length are given as functions of the bunch's head phase with respect to the coupler rf waveform. A phase of 90 degrf corresponds to the bunch head synchronized on the rf waveform crest.

\section{Implementing quadrupole correctors into the LCLS-II injector}

The LCLS-II injector consists of a $\mathrm{cw}$ rf gun, two solenoids for beam focusing, an rf buncher for bunch compression, and one standard eight-cavity cryomodule (CM) [23]. The standard eight-cavity CM is used to boost the beam energy to $\sim 100$ from $<1 \mathrm{MeV}$. The strong rotated quadrupole rf field located at the power coupler of the CM can increase the emittance, especially for a beam at low energy with a large beam size. Figure 11 shows the emittance simulations for a 300-pC bunch without coupler fields (blue line), with 3D coupler fields (green line), and with 3D coupler fields corrected by a quad corrector (red line). The coupler fields are computed from the 3D CAD model of the coupler's walls, ports, etc. The emittance is plotted vs $z$ along the length of the injector to where the emittance reaches its asymptotic value at $100 \mathrm{MeV}$. The emittance grows about $20 \%$ when the 3D rf coupler fields are included compared to the no coupler case. The simulation shows that a quad corrector located near the $\mathrm{CM}$ with $0.9 \mathrm{G}$ integrated quadrupole gradient and $-17.2^{\circ}$ rotation angle can eliminate the rf coupler's emittance growth (red vs green curves).

Because of these studies, we are planning to use a quad corrector in the second solenoid for both the anomalous quadrupole field and rf-coupler field correction in the LCLS-II injector [24]. We may consider installing another quad corrector near the entrance of the $\mathrm{CM}$ for the

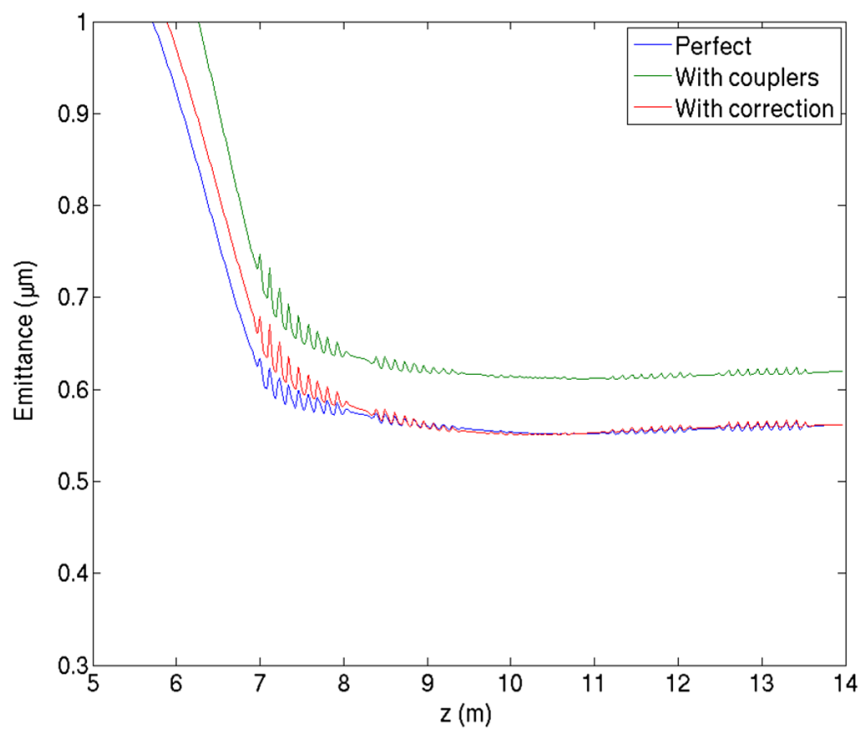

FIG. 11. LCLS-II injector emittance evolution with a perfect $\mathrm{rf}$ field (blue line), with 3D rf coupler fields (green line), and emittance cancellation using a weak $(0.9 \mathrm{G})$ rotated $\left(-17.2^{\circ}\right)$ correction quadrupole (red line) for $300 \mathrm{pC}$.

coupler correction if the shared quad corrector is not strong enough.

\section{SUMMARY AND CONCLUSIONS}

This paper discussed two types of emittance growth due to coupled transverse dynamics. These emittances are caused by rotated quadrupole fields in combination with a solenoid field and the rotated rf quadrupole fields of high power couplers for accelerator linacs. Both effects are commonly encountered in high brightness guns and injectors.

The emittance growth caused by a weak quadruple field in combination with a solenoidal field is analyzed for electron injectors. And its correction with a rotated quadrupole field is verified through analytical theory, simulations, and experiments. It is also shown that $\mathrm{rf}$ couplers in rf cavities can significantly degrade the emittance. Further theoretical analysis and simulations show that this rf-coupler emittance growth can also be completely corrected with a weak rotated quadrupole. These quadrupole correction techniques for solenoids and rf couplers have been implemented into the LCLS-I and LCLS-II injectors as well as in the SwissFEL injector [25] and the Cornell dc photocathode injector [26].

And finally, there is the potential use of rotated quadrupole correctors in highly dispersive systems such as chicane bunch compressors to correct for a small coupling of the transverse dynamics between the energy dispersed plane and the orthogonal, nondispersed plane dynamics due to slightly rotated dipoles. These and other examples demonstrate the general applicability of rotated corrector quadrupoles to uncouple the transverse dynamics of electron beams. 


\section{ACKNOWLEDGMENTS}

This work is supported by DOE under Grant No. DEAC02-76SF00515. The authors also acknowledge the good advice of the journal's anonymous referees. Their constructive comments and suggestions motivated us to transform and improve the final paper.

[1] G. Ripken, Report No. DESY RI-70/5, 1970.

[2] K. Wille, Report No. SLAC/AP-27, 1984.

[3] H. Qin, R. C. Davidson, M. Chung, and J. W. Burby, Generalized Courant-Snyder Theory for Charged-Particle Dynamics in General Focusing Lattices, Phys. Rev. Lett. 111, 104801 (2013).

[4] H. Wiedemann, Particle Accelerator Physics II, Nonlinear and Higher-Order Beam Dynamics (Springer, New York, 1995), Sec. 3.3.

[5] Z. Li, J. J. Bisognano, and B. C. Yunn, Transport Properties of the CEBAF Cavity, in Proceedings of the 15th Particle Accelerator Conference, PAC-1993, Washington, DC, 1993 (IEEE, New York, 1993) http://accelconf.web.cern.ch/ accelconf/p93/PDF/PAC1993_0179.PDF.

[6] Z. Li, Ph.D. dissertation, College of William and Mary, 1995.

[7] Z. Li, N. Folwell, L. Ge, A. Guetz, V. Ivanov, M. Kowalski, L.-Q. Lee, C. Ng, G. Schussman, L. Stingelin, R. Uplenchwar, M. Wolf, L. Xiao, and K. Ko, High performance computing in accelerating structure design and analysis, Nucl. Instrum. Methods Phys. Res., Sect. A 558, 168 (2006).

[8] L. Xiao, R. F. Boyce, D. H. Dowell, Z. Li, C. LimborgDeprey, and J. Schmerge, Dual feed RF gun design for the LCLS, in Proceedings of the 21st Particle Accelerator Conference, Knoxville, TN, 2005 (IEEE, Piscataway, NJ, 2005), http://accelconf.web.cern.ch/AccelConf/p05/ PAPERS/TPPE058.PDF.

[9] Z. Li, J. Chan, L. D. Bentson, D. H. Dowell, C. LimborgDeprey, J. Schmerge, D. Schultz, and L. Xiao, Coupler design of the LCLS injector S-band structures, in Proceedings of the 21st Particle Accelerator Conference, Knoxville, TN, 2005 (IEEE, Piscataway, NJ, 2005), http:// accelconf.web.cern.ch/AccelConf/p05/PAPERS/TPPT031 .PDF.

[10] D. C. Carey, K. L. Brown, and F. Rothacker, SLAC Report Nos. SLAC-R-530, Fermilab-Pub-98-310, and UC-414, pp. 148.

[11] K. T. McDonald, Expansion of an axially symmetric, static magnetic field in terms of its axial field, http://physics.princeton.edu/ mcdonald/examples/axial.pdf (unpublished).

[12] D. H. Dowell, Sources of emittance in $\mathrm{rf}$ photocathode injectors: Intrinsic emittance, space charge forces due to non-uniformities, arXiv:1610.01242.

[13] D. C. Carey, K. L. Brown, and F. Rothacker, SLAC Report Nos. SLAC-R-530, Fermilab-Pub-98-310, and UC-414, p. 127.
[14] D. H. Dowell, E. Jongewaard, J. Lewandowski, C. Limborg-Deprey, Z. Li, J. Schmerge, A. Vlieks, J. Wang, and L. Xiao, Report No. SLAC-Pub-13401, arXiv:1503.05877.

[15] D. C. Carey, K. L. Brown, and F. Rothacker, SLAC Report Nos. SLAC-R-530, Fermilab-Pub-98-310, and UC-414, p. 161.

[16] GPT: General Particle Tracer, version 2.82, Pulsar Physics, http://www.pulsar.nl/gpt/.

[17] R. Akre, D. Dowell, P. Emma, J. Frisch, S. Gilevich, G. Hays, Ph. Hering, R. Iverson, C. Limborg-Deprey, H. Loos, A. Miahnahri, J. Schmerge, J. Turner, J. Welch, W. White, and $\mathrm{J}$. $\mathrm{Wu}$, Commissioning the Linac Coherent Light Source injector, Phys. Rev. ST Accel. Beams 11, 030703 (2008).

[18] K. Floettmann, ASTRA manual, DESY, Germany, 2017, http://www.desy.de/ mpyflo/Astra_manual/Astra-Manual_ V3.2.pdf (unpublished).

[19] D. H. Dowell, SLAC Pubs Report No. LCLS-II-TN-15-05 [arXiv:1503.09142].

[20] M. Dohlus, I. Zagorodnov, E. Gjonaj, and T. Weiland, Coupler kick for very short bunches and its compensation, in Proceedings of the 11th European Particle Accelerator Conference, Genoa, 2008 (EPS-AG, Genoa, Italy, 2008), pp. 580-582, http://accelconf.web.cern.ch/AccelConf/e08/ papers/mopp013.pdf.

[21] Z. Li, F. Zhou, A. Vlieks, and C. Adolphsen, On the importance of symmetrizing RF coupler fields for low emittance beams, in Proceedings of the 24th Particle Accelerator Conference, PAC-2011, New York, 2011 (IEEE, New York, 2011), pp. 2044-2046, http://accelconf .web.cern.ch/AccelConf/PAC2011/papers/thoas1.pdf.

[22] M. S. Chae, J. H. Hong, Y. W. Parc, In Soo Ko, S. J. Park, H. J. Qian, W. H. Huang, and C.X. Tang, Emittance growth due to multipole transverse magnetic modes in an rf gun, Phys. Rev. ST Accel. Beams 14, 104203 (2011).

[23] LCLS-II Final Design Report, 2016, p. 49, https://docs .slac.stanford.edu/sites/pub/Publications/LCLSII\%20Final_ Design_Report.pdf.

[24] F. Zhou, D. Dowell, R. K. Li, T. O. Raubenheimer, J. Schmerge, C. Mitchell, C. Papadopoulos, F. Sannibale, and A. Vivoli, LCLS-II injector beamline design and RF coupler correction, in Proceedings of the 2015 International FEL Conference, Daejeon, Korea, http://accelconf.web .cern.ch/AccelConf/FEL2015/papers/mop021.pdf.

[25] T. Schietinger, M. Pedrozzi, M. Aiba, V. Arsov, S. Bettoni, B. Beutner, M. Calvi, P. Craievich, M. Dehler, F. Frei, R. Ganter, C. P. Hauri, R. Ischebeck, Y. Ivanisenko, M. Janousch, M. Kaiser, B. Keil, F. Löhl, G. L. Orlandi, C. Ozkan Loch, P. Peier, E. Prat, J.-Y. Raguin, S. Reiche, T. Schilcher, P. Wiegand, E. Zimoch, D. Anicic, D. Armstrong, M. Baldinger, R. Baldinger, A. Bertrand, K. Bitterli, M. Bopp, H. Brands, H. H. Braun, M. Brönnimann, I. Brunnenkant, P. Chevtsov, J. Chrin, A. Citterio, M. Csatari Divall, M. Dach, A. Dax, R. Ditter, E. Divall, A. Falone, H. Fitze, C. Geiselhart, M. W. Guetg, F. Hämmerli, A. Hauff, M. Heiniger, C. Higgs, W. Hugentobler, S. Hunziker, G. Janser, B. Kalantari, R. Kalt, Y. Kim, W. Koprek, T. Korhonen, R. Krempaska, M. 
Laznovsky, S. Lehner, F. Le Pimpec, T. Lippuner, H. Lutz, S. Mair, F. Marcellini, G. Marinkovic, R. Menzel, N. Milas, T. Pal, P. Pollet, W. Portmann, A. Rezaeizadeh, S. Ritt, M. Rohrer, M. Schär, L. Schebacher, St. Scherrer, V. Schlott, T. Schmidt, L. Schulz, B. Smit, M. Stadler, B. Steffen, L. Stingelin, W. Sturzenegger, D. M. Treyer, A. Trisorio, W. Tron, C. Vicario, R. Zennaro, and D. Zimoch,
Commissioning experience and beam physics measurements at the SwissFEL Injector Test Facility, Phys. Rev. Accel. Beams 19, 100702 (2016).

[26] A. Bartnik, C. Gulliford, I. Bazarov, L. Cultera, and B. Dunham, Operational experience with nanocoulomb bunch charges in the Cornell photoinjector, Phys. Rev. ST Accel. Beams 18, 083401 (2015). 